\title{
Mulheres em idade fértil: causas de internação em Unidade de Terapia Intensiva e resultados
}

\author{
Women of childbearing age: causes of \\ hospitalization in intensive care unit and results
}

Cátia Millene Dell Agnolo ${ }^{1}$, Angela Andréia França Gravena², Tiara Cristina Romeiro-Lopes², Sheila Cristina Rocha-Brischiliari², Maria Dalva de Barros Carvalho², Sandra Marisa Pelloso²

1Programa de Pós-Graduação em Enfermagem da Universidade Estadual de Maringá (UEM) - Maringá (PR), Brasil.

2Programa de Pós-Graduação em Ciências da Saúde da UEM - Maringá (PR), Brasil.

DOI: http://dx.doi.org/10.7322/abcshs.v39i2.626

\section{RESUMO}

Introdução: Pacientes obstétricas representam uma fração significativa das admissões em unidades de cuidado intensivo e consistem em um desafio para a equipe. Objetivo: Analisar as principais causas de internação e morte materna de mulheres em idade fértil ocorridas em unidades de terapia intensiva de hospitais de um município do Noroeste do Paraná, Sul do Brasil. Métodos: Estudo exploratório, descritivo, retrospectivo, realizado com mulheres em idade fértil (10 a 49 anos) internadas em três unidades de terapia intensiva existentes em um município do Noroeste do Paraná, por causas obstétricas e não obstétricas, no período de janeiro de 2005 a dezembro de 2009. Resultados: Foram encontradas 775 internações de mulheres em idade fértil, com uma média de idade de $33,7 \pm 10,3$ anos, com duração média de internação de $5,0 \pm 8,4$ dias. A maior parte das mulheres era do município estudado $(67,2 \%)$, com baixa escolaridade (nível primário, 68,2\%). Entre as causas obstétricas de internação, a pré-eclâmpsia grave e a eclâmpsia se constituíram na principal causa (71,3\%); a pielonefrite se destacou sob outras condições no período gestacional (53\%). Não foi encontrada associação estatística entre internações por causas obstétricas em unidade de terapia intensiva e óbito. Conclusão: As causas não obstétricas representaram a maioria das internações de mulheres em idade fértil nas unidades de terapia intensiva do município, no período estudado e, entre as causas obstétricas, a pré-eclâmpsia grave e a eclâmpsia constituíram a principal causa.

Palavras-chave: unidades de terapia intensiva; saúde da mulher; período fértil.

\begin{abstract}
Introduction: Obstetric patients represent a significant fraction of admissions to intensive care units and consist of a challenge to the team. Objective: To analyze the main causes of maternal death and hospitalization of women of childbearing age occurring in intensive care units of hospitals in a city in Northwestern Paraná, Southern Brazil. Methods: Exploratory, descriptive and retrospective study, conducted with women of childbearing age (10-49 years) admitted in three intensive care units existing in a city of Northwest Paraná, for obstetric and non-obstetric causes, from January 2005 to December 2009. Results: There were 775 admissions of women of childbearing age, with a mean age of $33.7 \pm 10.3$ years; the mean duration of hospitalization was $5.0 \pm 8.4$ days. Most of the women were from the municipality $(67.2 \%)$, with low education level (primary level, $68.2 \%$ ). Among the obstetric causes of hospitalization, pre-eclampsia and eclampsia constituted the main cause (71.3\%); pyelonephritis stood out other conditions during pregnancy (53\%). There was no statistical association between hospital admissions for obstetric causes in intensive care unit and death. Conclusion: Non-obstetric causes accounted for the majority of hospitalizations of women of childbearing age in the intensive care unit of this municipality during the study period; among obstetric causes, pre-eclampsia and eclampsia were the main causes.
\end{abstract}

Keywords: intensive care units; women's health; fertile period.

Recebido em: 22/07/2013

Revisado em: 14/01/2014

Aprovado em: 14/04/2014 


\section{INTRODUÇÃO}

A internação de pacientes com causas obstétricas em unidades de terapia intensiva (UTI) consiste em um desafio para a equipe de cuidados intensivos ${ }^{1}$. A gravidez, com suas alterações fisiológicas específicas, diferencia as pacientes em relação aos demais atendimentos em UTI, agravando condições clínicas, além de afetar a interpretação de testes diagnósticos e laboratoriais².

Segundo dados da Organização Mundial da Saúde (OMS), em 2005 ocorreram mais de 500 mil mortes maternas, 95\% delas em países em desenvolvimento ${ }^{3}$, sendo um indicador importante de morbidade materna a taxa de transferência de mulheres no período gravídico-puerperal para uma UTI, em uma determinada região específica ${ }^{4}$.

As chances de admissões em UTI de mulheres durante o ciclo gravídico puerperal são maiores do que as de mulheres não grávidas².

Aproximadamente $90 \%$ dos partos no Brasil ocorrem em hospitais e $67,1 \%$ das mortes são consequência de causas obstétricas diretas, representadas principalmente por transtornos hipertensivos (25\% dos casos) e doença hipertensiva específica da gravidez (37\%). As causas obstétricas indiretas respondem por um quarto do total ${ }^{5}$.

Os dados relativos à mortalidade de pacientes com causas obstétricas internadas em UTI apresentam grandes variações (0 a 40\% $)^{6}$. No entanto, a comparação entre os setores de UTI em diversos hospitais é bastante prejudicada, pois cada setor apresenta diferentes especificidades de atendimento e clientelas específicas. Não obstante, há ainda uma diferença considerável entre as taxas de mortalidade materna nos países desenvolvidos e nos países em desenvolvimento, sendo, nesse último, superiores ${ }^{7}$. Além disso, os dados sobre as características epidemiológicas dessas pacientes são escassos. Na América do Sul, por exemplo, existem poucos estudos voltados a essa clientela ${ }^{8-11}$.

Partindo do pressuposto da necessidade de se conhecer dados epidemiológicos sobre internações obstétricas, morbidade e mortalidade dessas pacientes em UTI, foi realizado o presente estudo, com o objetivo de analisar as principais causas de internação e morte materna de mulheres em idade fértil ocorridas em UTI de hospitais de um município do Noroeste do Paraná, Sul do Brasil.

\section{MÉTODOS}

Trata-se de um estudo descritivo, exploratório, retrospectivo, realizado com mulheres em idade fértil (10 a 49 anos) internadas em três UTI existentes em um município do Noroeste do Paraná, por causas obstétricas e não obstétricas, durante o período de janeiro de 2005 a dezembro de 2009. A coleta de dados foi realizada em um hospital ensino, um particular e um filantrópico/particular.

O hospital ensino disponibilizava oito leitos de terapia intensiva, o hospital particular apresentava seis leitos para atendimento e o filantrópico/particular, nos primeiros anos de estudo, contava com 12 leitos de atendimento, sendo que no último ano teve seu número de leitos aumentado para 22. Todas estas UTI são de atendimento a pacientes gerais e dispõem de ventiladores mecânicos para todos os leitos. Os médicos trabalham em regime de plantão de 24 horas, sendo que todos os hospitais possuem enfermeiro específico no setor nos períodos diurno e noturno e, apenas no hospital particular (seis leitos), o enfermeiro do período noturno oferece cobertura ao setor de pronto atendimento do hospital.

Os três hospitais contavam com o suporte de especialistas como neurologistas, nefrologistas, entre outros, e possibilitavam a realização de procedimentos de imagem (radiografia, ultrassonografia e tomografia computadorizada) nas dependências da própria instituição.

As variáveis analisadas foram: idade (em anos), procedência, estado conjugal, escolaridade, história obstétrica (época de internamento: fase do ciclo gravídico puerperal, paridade, idade gestacional do parto), diagnóstico médico de entrada, duração do internamento e evolução/desfecho (alta da UTI ou óbito).

A coleta de dados foi realizada através de consulta em livro registro de internamentos de cada UTI e coleta de dados referentes às variáveis. Esse livro é preenchido por profissional secretário, enfermeiro ou técnico de enfermagem do setor e engloba dados diversos relativos à internação, podendo ser preenchido parcialmente na ausência de dados à entrada da paciente no setor, sendo completado posteriormente. A evolução do internamento é preenchida num segundo momento, mediante alta, transferência ou óbito.

$\mathrm{Na}$ análise dos dados foram utilizados os programas Microsoft Excel e Statística 8.0, sendo apresentados na forma de tabela de distribuição de frequência para as variáveis categóricas. Foram calculados média e desvio padrão para as variáveis quantitativas. O teste do $\chi^{2}$ foi utilizado para verificação de associação entre as variáveis, com significância de $5 \%$.

Após autorização das instituições participantes, a pesquisa foi apreciada e aprovada pelo Comitê de Ética da Universidade Estadual de Maringá, mediante o Parecer 017/2009. Foi solicitada ao presente Comitê de Ética, a liberação da assinatura do Termo de Consentimento Livre e Esclarecido, por se tratar de dados indiretos, provenientes de livros de registros.

\section{RESULTADOS}

Houve 775 internações de mulheres em idade fértil no período de cinco anos nas UTI estudadas, com uma média de idade $(n=771)$ de 33,7 $\pm 10,3$ anos, variando de 12 a 49 anos.

De um total de 393 mulheres, 67,2\% ( $n=264)$ foram procedentes do município estudado e 32,3\% (127) eram de outros municípios do Paraná.

Aproximadamente metade, 50,8\% (151), não possuíam companheiro $(n=297)$ e de um total de 146 mulheres a respeito das quais constava a profissão, a maior parte $(69,8 \%)$ era do lar $(n=102)$, seguida por estudante $(24,7 \%, \mathrm{n}=36)$. 
A escolaridade baixa prevaleceu. Entre 129 mulheres, $88(68,2 \%)$ possuíam nível primário; 6,2\% (8), nível secundário; e $16,3 \%$ (21), nível superior.

A maior parte da internação foi pelo Sistema Único de Saúde (SUS) e convênios particulares, os quais podem ser vistos da Tabela 1, assim como a evolução final do internamento na UTI. A distribuição das causas de internação podem ser vistas na Tabela 2. A prevalência da mortalidade por causas obstétricas foi de $7,3 \%$, e nas demais pacientes internadas por causas não obstétricas, a prevalência foi de $17,2 \%$.

Entre os anos estudados (2005 a 2009) foram identificadas $96(12,4 \%)$ internações de mulheres em idade fértil por causas obstétricas e 679 (87,6\%) internações por causas não obstétricas.

De um total de 124 óbitos ocorridos no período do estudo, 7 (5,6\%) foram relacionados a causas obstétricas e $117(94,4 \%)$ a causas não obstétricas.

Não foi encontrada associação estatística entre internações por causas obstétricas em UTI e óbito (valor $\mathrm{p}=0,284$ ).

Quanto à duração da internação $(\mathrm{n}=720)$, a média foi de $5 \pm 8,4$ dias, variando de 1 a 74 dias.

\section{DISCUSSÃO}

Apesar de ser um grupo pequeno, as pacientes com causas obstétricas representam um importante grupo de pacientes em UTI, pois requerem cuidados especializados e apresentam alterações próprias, fisiológicas da gravidez, as quais podem confundir as tomadas de decisão ${ }^{12}$. A gravidez, com suas alterações fisiológicas específicas, diferenciam as pacientes em relação aos demais atendimentos em UTI, agravando condições clínicas, além de afetar a interpretação de testes diagnósticos e laboratoriais².

Tratamento intensivo adequado às pacientes com causas obstétricas instáveis representou diminuição significativa da mortalidade materna na última década ${ }^{12,13}$.

Despreparo dos profissionais no manejo precoce de situações graves nessa população específica, dificuldades de acesso à rede básica, falhas no sistema de referência e contra-referência, dificultando o acesso à assistência mais complexa representam alguns dos fatores contribuintes para um desfecho desfavorável nesses $\operatorname{casos}^{14}$.

Portanto, os profissionais que atuam em UTI devem possuir experiência em assuntos relacionados à obstetrícia e terapia intensiva para a prestação de cuidados a essas pacientes. Dessa forma, conhecer as principais causas de internação dessas mulheres em UTI pode auxiliar no preparo e na formação dos profissionais que atuam nesses setores, visto que, em nossa região, não são comuns UTI específicas para atendimento em obstetrícia.

A mortalidade materna, ou seja, a morte da mulher durante a gravidez ou dentro de 42 dias completos do puerpério, consiste em uma das principais avaliações das condições sociais de um país. Pode ser considerada, ainda, um excelente indicador de saúde, não somente da mulher, mas de toda a população ${ }^{15}$.
Tabela 1: Distribuição das mulheres em idade fértil internadas em unidade de terapia intensiva segundo convênio e evolução, no período de janeiro de 2005 a dezembro de 2009, Maringá, Paraná, Brasil, 2009

\begin{tabular}{|l|c|c|}
\hline Variáveis & $\mathbf{n = 7 7 5}$ & $\%$ \\
\hline Evolução & & \\
\hline Alta & 610 & 78,8 \\
\hline Alta a pedido & 1 & 0,1 \\
\hline Transferência & 124 & 1,5 \\
\hline Óbito & 28 & 16,0 \\
\hline $\begin{array}{l}\text { Ignorado } \\
\text { Convênio }\end{array}$ & 3,6 \\
\hline $\begin{array}{l}\text { Particulares/tabela } \\
\text { Seguro }\end{array}$ & 19 & 4,4 \\
\hline SUS/gratuito* & 376 & 48,5 \\
\hline $\begin{array}{l}\text { Outros convênios } \\
\text { Ignorado }\end{array}$ & 345 & 44,5 \\
\hline Característica atendimento Hospital & 1 & 0,1 \\
\hline $\begin{array}{l}\text { SUS } \\
\text { Particular e convênios }\end{array}$ & 141 & 18,2 \\
\hline $\begin{array}{l}\text { Filantrópico, SUS, particulares e } \\
\text { convênios }\end{array}$ & 163 & 21,0 \\
\hline
\end{tabular}

*Dois atendimentos registrados como gratuitos; SUS: Sistema Único de Saúde

Tabela 2: Distribuição das causas obstétricas de mulheres em idade fértil internadas em unidade de terapia intensiva, no período de janeiro de 2005 a dezembro de 2009, Maringá, Paraná, Brasil, 2009

\begin{tabular}{|c|c|c|}
\hline \multirow{2}{*}{$\begin{array}{l}\text { Anos } \\
\text { Variáveis }\end{array}$} & \multicolumn{2}{|c|}{2005 a 2009} \\
\hline & $\mathrm{n}=96$ & $\%$ \\
\hline \multicolumn{3}{|l|}{ Causas obstétricas ( $n=42 ; 43,8 \%$ ) } \\
\hline Eclâmpsia/pré-eclâmpsia grave & 30 & 71,3 \\
\hline Hellp síndrome & 7 & 16,7 \\
\hline Gravidez ectópica & 2 & 4,8 \\
\hline Trabalho de parto prematuro & 2 & 4,8 \\
\hline Mola hidatiforme & 1 & 2,4 \\
\hline \multicolumn{3}{|l|}{$\begin{array}{l}\text { Outras causas/período gestacional }(n=17 \text {; } \\
17,7 \%)\end{array}$} \\
\hline Causas cardíacas/respiratórias & 5 & 29,4 \\
\hline Sepse (aborto) & 3 & 17,6 \\
\hline Pielonefrite & 9 & 53,0 \\
\hline \multicolumn{3}{|l|}{ Após cesariana $(n=28 ; 29,1 \%)$} \\
\hline $\begin{array}{l}\text { Síndromes } \\
\text { (Ogilvie e Wolf Parkinson White) }\end{array}$ & 2 & 2,1 \\
\hline Sepse & 3 & 3,1 \\
\hline Sistema respiratório/cardíaco & 5 & 5,2 \\
\hline Histerectomia e causas relacionadas & 10 & 10,4 \\
\hline Anemia/hemorragia & 7 & 7,3 \\
\hline Descolamento prematuro placenta & 1 & 1,0 \\
\hline $\begin{array}{l}\text { Outras causas não relacionadas à } \\
\text { gestação* }(n=9 ; 9,4 \%)\end{array}$ & 9 & 9,4 \\
\hline
\end{tabular}

*pós-operatório ooforectomia (3); doença inflamatória pélvica (2); abscesso ovariano (2); neoplasia de mama (1); causa não especificada (1)

Cerca de 32,8\% das mulheres estudadas eram provenientes de outros municípios vizinhos e/ou estados. O município onde foi realizado o estudo serve como referência aos municípios vizinhos e, dois dos hospitais estudados são referência ao tratamento da 
gestante de alto risco e possuem terapia intensiva neonatológica para atendimento dos recém-nascidos.

Quase metade $(49,2 \%)$ das mulheres estudadas possuíam companheiro. Em estudo realizado na cidade de Chapecó, Santa Catarina, o índice de mulheres com o mesmo perfil de estudo e que possuíam companheiro (casadas, uniões livres) foi de $70 \%{ }^{16}$.

Escolaridade baixa foi descrita por outros autores nessa mesma população ${ }^{16}$. Em nosso estudo, mulheres com nível primário representaram $68,2 \%$ das estudadas.

A média de idades encontrada em nosso estudo foi de 33,7 anos. Estudos com idade média semelhante foram encontrados ${ }^{13,17,18}$, bem como com idade inferior $(25,5 \pm 4,6$ anos), como em um estudo realizado na Índia com 453 mulheres internadas em UTI por causas obstétricas ${ }^{19}$.

Num estudo realizado em La Plata, Província de Buenos Aires, Argentina, durante o período entre 01 de janeiro de 1998 e 30 de setembro de 2005, 10\% de todas as admissões em UTI eram de pacientes com causas obstétricas. Destas, 102 foram internadas no pós parto e 59 durante a gravidez ${ }^{8}$.

Pacientes obstétricas representam uma fração significativa das admissões em unidades de cuidado intensivo em países onde a mortalidade é elevada, como a Índia, que apresenta 548 admissões a cada 100 mil nascimentos e taxa de mortalidade materna em torno de $21,6 \%{ }^{19}$.

Em um hospital de Forças Armadas da Arábia Saudita, um estudo descreveu uma porcentagem de 1,6\% de admissões obstétricas entre todas as admissões em UTI, sendo que 76\% delas foi após cesariana e as principais indicações de admissão foram hemorragia e hipertensão ${ }^{13}$.

Neste estudo foram identificadas 775 mulheres em idade fértil admitidas em UTI, sendo que as causas obstétricas ficaram em terceiro lugar em número de internamentos, sendo responsáveis por $12,3 \%$ das internações. Entre as causas obstétricas, os transtornos hipertensivos foram responsáveis por $71,3 \%$ dos casos e entre todas as causas, representaram $31,3 \%$ (dado não informado). Outros autores descreveram dados similares, onde os transtornos hipertensivos (eclâmpsia) representaram $66 \%$ das admissões em $\mathrm{UTI}^{16}$. As hemorragias totalizaram 9,4\% das causas após cesariana e 7,3\% de todas as causas de internação de mulheres em idade fértil (dado não informado). Internações após cesariana totalizaram $29,1 \%$ das internações.

Estas mesmas causas, transtornos hipertensivos e hemorragias obstétricas severas, foram as principais causas de internação em UTI segundo outros estudos ${ }^{13,16,20}$.

Em relação às síndromes hipertensivas, em vários outros estudos também foram identificadas como uma das causas mais importantes associadas à morbidade materna grave ${ }^{10,21-25}$, inclusive em um estudo de revisão sistemática da literatura entre os anos de 1990 e 2008, onde as síndromes hipertensivas foram as mais prevalente em UTI não havendo diferença entre países desenvolvidos e em desenvolvimento ${ }^{6}$. Em nossa pesquisa, cerca de $71,3 \%$ das mulheres internaram por pré-eclâmpsia grave e eclâmpsia.
Se associarmos a Síndrome de Hellp (16,7\%), totalizaremos 88,0\% das internações por essas causas. Esse fato chama a atenção para a necessidade de priorizar a prevenção e o tratamento precoce desses agravos, bem como otimizar o conhecimento dos profissionais que trabalham no setor e em terapia intensiva.

Hemorragia grave, em nosso estudo, foi verificada em 19,1\% das mulheres, o que está próximo a valores encontrados por outros autores ${ }^{24}$. Outro estudo, realizado com 98 maternidades holandesas, revelou 48,6\% das internações por hemorragias obstétricas ${ }^{26}$.

Apesar de descrever um índice elevado de síndromes hipertensivas $(67,7 \%)$ como principal causa de internação em UTI, outro estudo descreve as hemorragias como principal causa de mortalidade nesse mesmo setor ${ }^{27}$.

A mortalidade de mulheres em idade fértil com internação em UTI por causas obstétricas em nosso estudo foi de 7,3\%, inferior à mortalidade por causas não obstétricas, a qual foi de $17,2 \%$. Em um estudo com número menor de internações, o índice encontrado de mortalidade foi de uma morte para dez internações (10\%), superior aos nossos dados ${ }^{16}$.

As internações duraram em média cinco dias em nosso estudo, com $78,8 \%$ de alta e $16 \%$ de óbito, ao todo, entre as mulheres estudadas, internadas por causas obstétricas ou não, superior a outro estudo, realizado com 43 mulheres internadas por causas obstétricas, que foi de 3 dias $^{17}$. Internações por período inferior foram descritas em outros estudos, com média de hospitalização de 1,6 dias ${ }^{16}$ e dois dias ${ }^{13}$. Há que se investigar a indicação precoce e as causas médicas de internação como explicação para essa variação de período.

Além disso, a disponibilidade de leitos e os critérios de indicação para a internação em UTI podem não ser uniformes, por tratar-se de vários serviços avaliados, tornando esse marcador questionável, uma vez que pode ser influenciado pela complexidade, número de vagas e organização da rede de assistência obstétrica ${ }^{28,29}$.

A falta de atendimento pré-natal e a demora na indicação de UTI são fatores agravantes e danosos às pacientes com causas obstétricas, porém, evitáveis por prevenção ${ }^{19}$, sendo que esse fato não foi verificado nesta pesquisa.

Outro estudo realizado para, entre outros objetivos, avaliar as causas não obstétricas de internação em UTI encontrou uma porcentagem de $9,8 \%^{30}$. Dado similar ao encontrado em nosso estudo $(9,4 \%)$.

Há algumas limitações neste estudo a serem consideradas: os dados são retrospectivos e foram coletados manualmente em livros de registro de internamento, impossibilitando o aprofundamento de questões relacionadas ao tratamento e à assistência prestados. Por este motivo não foi possível avaliá-los segundo critérios near miss ou mesmo morbidade severa grave, como a maioria dos trabalhos efetuados com esse mesmo tema. Os dados referentes à evolução/mortalidade se referem tão somente ao período de internação em UTI, não considerando os dados de mortalidade tardia, que podem ter ocorrido. A casuística é 
pequena, embora tenham sido verificados cinco anos e dois estabelecimentos que são referência para o atendimento de obstetrícia para o município e um deles é referência regional para essa mesma especialidade, englobando o atendimento a mais de 26 municípios.
No entanto, pode e deve servir de base para organização de estudos futuros para monitoramento desses casos, bem como avaliação do desempenho desses serviços de obstetrícia e UTI que oferecem suporte para atendimento às pacientes de maior gravidade, pois estudos desta natureza são escassos em nossa região.

\section{REFERÊNCIAS}

1. Price LC, Germain S, Wyncoll D, Nelson-Piercy C. Management of the critically ill obstetric patient. Obstet Gynaecol Reprod Med. 2009;19(12):350-8. http://dx.doi.org/10.1016/j.ogrm.2009.09.004

2. Amorim $M M R$, Katz $L$, Ávila $M B$, Araújo $D E$, Valença $M$, Albuquerque CJM, et al. Perfil das admissões em uma unidade de terapia intensiva obstétrica de uma maternidade brasileira. Rev Bras Saúde Mater Infant. 2006;6(Suppl 1):s55-62. http://dx.doi.org/10.1590/S1519-38292006000500008.

3. World Health Organization (WHO) [Internet]. World Health Statistics 2009. Disponível em: http://www.who.int/whosis/whostat/ EN_WHSO9_Full.pdf. Acesso em: 10 jan. 2010.

4. Mahutte NG, Murphy-Kaulbeck L, Le Q, Solomon J, Benjamin A, Boyd A. Obstetric admissions to the intensive care unit. Obstet Gynecol. 1999;94(2):263-6. http://dx.doi.org/10.1016/S0029-7844(99)00274-4

5. Amaral E, Luz AG, Souza JPD. A morbidade materna grave na qualificação da assistência: utopia ou necessidade? Rev Bras Ginecol Obstetr. 2007;29(9):484-89 http://dx.doi.org/10.1590/S0100-72032007000900008

6. Pollock W, Rose L, Dennis CL. Pregnant and postpartum admissions to the intensive care unit: a systematic review. Intensive Care Med. 2010;36(9):1465-74 http://dx.doi.org/10.1007/s00134-010-1951-0

7. Thonneau PF, Matsudai T, Alihonou E, Souza J, Faye O, Moreau JC, et al. Distribution of causes of maternal mortality during delivery and post-partum: results of an African multicentre hospital-based study. Eur J Obstet Gynecol Reprod Biol. 2004:114(2):150-4.

http://dx.doi.org/10.1016/j.ejogrb.2003.12.004

8. Vasquez DN, Estenssoro E, Canales HS, Reina R, Saenz MG, Neves AV, et al. Clinical characteristics and outcomes of obstetric patients requiring ICU admission. Chest. 2007;131(3):718-24. http://dx.doi.org/10.1378/chest.06-2388

9. Souza JP, Cecatti JG, Parpinelli MA, Sousa MH, Serruya SJ. Revisão sistemática sobre morbidade materna near miss. Cad Saúde Pública. 2006;22(2):255-64. http://dx.doi.org/10.1590/S0102-311X2006000200003

10. Souza JP, Cecatti JG. The near-miss maternal morbidity scoring system was tested in a clinical setting in Brazil. J Clin Epidemiol. 2005;58(9):962.

http://dx.doi.org/10.1016/j.jclinepi.2005.02.011

11. Amaral E, Souza JP, Surita F, Luz AG, Sousa MH, Cecatti JG, et al. A population-based surveillance study on severe acute maternal morbidity (near-miss) and adverse perinatal outcomes in Campinas, Brazil: the Vigimoma Project. BMC Pregnancy Childbirth. 2011;11:9. http://dx.doi.org/10.1186/1471-2393-11-9

12. Amorim MMR, Katz L, Santos LC. Terapia intensiva em obstetrícia. Femina. 2007;35(2):107-11.

13. Anwari JS, Butt AA, Al-Dar MA. Obstetric admissions to the intensive care unit. Saudi Med J. 2004;25(10):1394-9.
14. Geller SE, Adams MG, Kominiarek MA, Hibbard JU, Endres LK, Cox SM, et al. Reliability of a preventability model in maternal death and morbidity. Am J Obstet Gynecol. 2007;196(1):57.e1-6. http://dx.doi.org/10.1016/j.ajog.2006.07.052

15. Laurenti R, Jorge MHPM, Gotlieb SLD. A mortalidade materna nas capitais brasileiras: algumas características e estimativa de um fator de ajuste. Rev Bras Epidemiol. 2004:7(4):449-60. http://dx.doi.org/10.1590/S1415-790X2004000400008

16. Cecconelo F, Ferraz L. O perfil sócio-demográfico e patológico de gestantes e puérperas admitidas na UTI de um hospital do Oeste Catarinense. Rev Divulg Cient. 2010;17(1):71-8.

17. Cheng C, Raman S. Intensive care use by critically ill obstetric patients: a five-year review. Int J Obstet Anesth. 2003;12(2):89-92.

http://dx.doi.org/10.1016/S0959-289X(02)00154-1

18. Gilbert TT, Smulian JC, Martin AA, Ananth CV, Scorza W, Scardella AT. Obstetric admissions to the intensive care unit: outcomes and severity of illness. Obstet Gynecol. 2003;102(5 Pt 1):897-903. http://dx.doi.org/10.1016/S0029-7844(03)00767-1

19. Karnad DR, Lapsia V, Krishnan A, Salvi VS. Prognostic factors in obstetric patients admitted to an Indian intensive care unit. Crit Care Med. 2004;32(6):1294-9. http://dx.doi.org/10.1097/01.CCM.0000128549.72276.00

20. Zeeman GG. Obstetric critical care: a blueprint for improved outcomes. Crit Care Med. 2006;34(9 Suppl):S208-14. http://dx.doi.org/10.1097/01.CCM.0000231884.99763.69

21. Amorim MMR, Katz L, Valença M, Araújo DE. Morbidade materna grave em UTI obstétrica no Recife, região nordeste do Brasil. Rev Assoc Med Bras. 2008:54(3):261-6. http://dx.doi.org/10.1590/S0104-42302008000300021

22. Luz AG, Tiago DB, Silva JC, Amaral E. Severe maternal morbidity at a local reference university hospital in Campinas, São Paulo, Brazil. Rev Bras Ginecol Obstet. 2008;30(6):281-6. http://dx.doi.org/10.1590/S0100-72032008000600003

23. Souza JP, Cecatti JG, Parpinelli MA, Serruya SJ, Amaral E. Appropriate criteria for identification of near-miss maternal morbidity in tertiary care facilities: a cross sectional study. BMC Pregnancy Childbirth. 2007;11(7):20 http://dx.doi.org/10.1186/1471-2393-7-20

24. Morse ML, Fonseca SC, Gottgtroy CL, Waldmann CS, Gueller E. Morbidade materna grave e Near Misses em Hospital de Referência Regional. Rev Bras Epidemiol. 2011;14(2):310-22. http://dx.doi.org/10.1590/S1415-790X2011000200012

25. Orsini J, Butala A, Diaz L, Muzylo E, Mainardi C, Kastell P. Clinical profile of obstetric patients admitted to the Medical-Surgical Intensive Care Unit (MSICU) of an Inner-City Hospital in New York. J Clin Med Res. 2012;4(5):314-7. http://dx.doi.org/10.4021/Fjocmr1079w

26. Zwart JJ, Dupuis JR, Richterns A, Ory F, van Roosmalen J. Obstetric intensive care unit admission: a 2-year nationwide populationbased cohort study. Intensive Care Med. 2010; 36(2):256-63. http://dx.doi.org/10.1007/s00134-009-1707-x 
27. Lotufo FA, Parpinelli MA, Haddad SM, Surita FG, Cecatti JG. Applying the new concept of maternal near-miss in an intensive care unit. Clinics. 2012;67(3):225-30. http://dx.doi.org/10.6061/clinics/2012(03)04

28. Sousa MH, Cecatti JG, Hardy EE, Serruya SJ. Severe maternal morbidity (near miss) as a sentinel event of maternal death. An attempt to use routine data for surveillance. Reprod Health. 2008;5:6

http://dx.doi.org/10.1186/1742-4755-5-6
29. Adisasmita A, Deviany PE, Nandiaty F, Stanton C, Ronsmans C. Obstetric near miss and deaths in public and private hospitals in Indonesia. BMC Pregnancy Childbirth. 2008;8:10. http://dx.doi.org/10.1186/1471-2393-8-10

30. Coelho MAL, Katz L, Coutinho I, Hofmann A, Miranda L, Amorim M. Perfil de mulheres admitidas em uma UTI obstétrica por causas não obstétricas. Rev Assoc Med Bras. 2012;58(2):160-7.

http://dx.doi.org/10.1590/S0104-42302012000200011 